\title{
Constraints on the weak r-process: Abundance of Palladium in metal poor stars
}

\section{Patrick François*}

Paris-Meudon Observatory, France \& Université de Picardie Jules Verne, Amiens, France

E-mail: patrick.francoiseobspm.fr

\section{S. Wanajo}

TUM / MPA Garching, Germany

\section{N. Prantzos}

IAP, Paris, France

G. James

ESO, Garching, Germany

Y. Ishimaru

ICU, Mitaka, Tokyo, Japan

\section{W. Aoki}

NAOJ, Mitaka-Tokyo, Japan

\section{S. Honda}

Kyoto University, Japan

\begin{abstract}
A french-japanese collaboration has permitted to observe 3 exceptionally rare stars thanks to spectra obtained at the Subaru telescope in Hawaii and the VLT in Paranal. These stars are not only extremely metal poor stars but exhibit a very high $[\mathrm{Sr} / \mathrm{Ba}]$ ratio. The aim of this project was to detect and measure the $3404.579 \AA$ transition of Palladium, an element with an atomic number between $\mathrm{Sr}$ and $\mathrm{Ba}$. One true measurement and two upper limits of Palladium, combined to the data found in the literature support the hypothesis of a correlation between $[\mathrm{Pd} / \mathrm{Ba}]$ and $[\mathrm{Sr} / \mathrm{Ba}]$ supporting the hypothesis that Pd is substancially produced by weak r-process during the early phases of the galactic evolution.
\end{abstract}

11th Symposium on Nuclei in the Cosmos

19-23 July 2010

Heidelberg, Germany.

\footnotetext{
*Speaker.
} 


\section{Introduction}

Many sites have been proposed to explain the creation of the r-process elements. All these sites have in common that the r-process elements (typically europium) have been formed under dynamical conditions and associated with the final stages of evolution of massive stars or binary compact mergers. This process is a primary process, i.e. it does not need to have $\mathrm{Fe}$ already present in the star to form the r-process. As a consequence, SNeII or mergers with no or little Fe content are able to build r-process but not s-process elements. Therefore, the heavy elements that we see in very metal poor stars have been only built by r-process. This point has been made already by [11].

The variation of the ratios $[$ Heavy-element/Fe] vs $[\mathrm{Fe} / \mathrm{H}]$ measured in metal poor stars can be used to constrain the different sites of r-process production. (see for example [10] and reference therein.)

If all the heavy elements are formed by a unique source (single site) of r-process synthesis, therefore abundance ratios of heavy elements (like [Eu/Ba]) should be constant in very metal poor stars of different metallicities. This is indeed what is found for many of the heavy elements like $\mathrm{Ba}, \mathrm{Eu}, \mathrm{La}, \mathrm{Ce}, \ldots . .$.

However, the large dispersion found in $[\mathrm{Sr} / \mathrm{Ba}]$ ratios as a function of $[\mathrm{Fe} / \mathrm{H}]$ suggests that $\mathrm{Sr}$ at least does not come from a universal process. Furthermore, abundance patterns of several metalpoor stars show that lighter neutron-capture elements $(Z<56)$ significantly deviate from the scaled solar r-process curve (e.g., [5]; [9]. These observations imply the presence of another astrophysical site for lighter r-process elements.

For example, theoretical calculations on r-process nucleosynthesis by [12] and chemical evolution of the Galaxy by [6] suggests the existence of a process favoring the synthesis of the "lighter" heavy elements ( $\mathrm{Sr}, \mathrm{Y}, \mathrm{Zr}$ ), called the "weak r-process", a process which will form the light heavyelements and not the heavier ones. The site of this process would be associated with SN explosions from the collapse of Ne-O-Mg cores [13]. In order to investigate the extent (in atomic number) of the second r-process, we focused on the abundance of $\mathrm{Pd}$, an element with an atomic number between $\mathrm{Sr}$ and $\mathrm{Ba}$, in stars with large excesses of light neutron-capture elements (e.g., $\mathrm{Sr}, \mathrm{Z}=38$ ). Theoretical calculations have predicted a tight correlation between $[\mathrm{Pd} / \mathrm{Ba}]$ and $[\mathrm{Sr} / \mathrm{Ba}]$ if $\mathrm{Pd}$ is mostly built by weak r-process during the early galactic phase [6].

\section{Observations}

The observations have been performed using SUBARU + HDS for the northern star and Kueyen + UVES for the two southern targets. The cumulative exposure time for the 3 stars exceeds 20 hours. A classical LTE analysis has been carried out for our sample of stars. We made use of the OSMARCS model atmospheres. Solar abundances were adopted from [4]. The elemental abundance analysis was carried out using a current version of the turbospectrum code [1] 


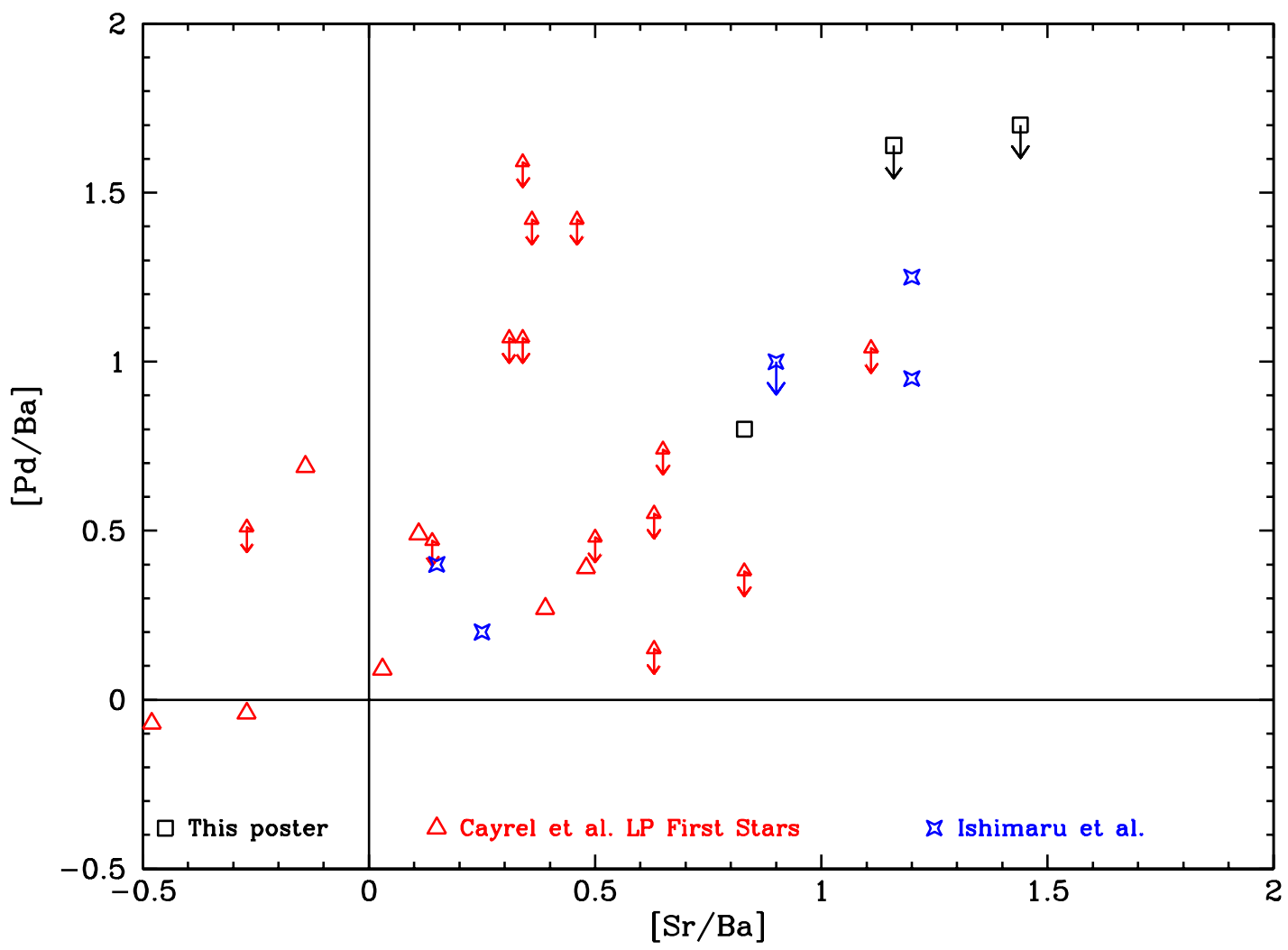

Figure 1: $[\mathrm{Pd} / \mathrm{Ba}]$ as a function of $[\mathrm{Sr} / \mathrm{Ba}]$. Black squares : data from this paper. Red triangles : data from Cayrel et al. LP First Stars (priv. comm.). Triangles with arrows represent upper limits. Blue stars : data from Ishimaru et al. (2004).

\begin{tabular}{lcccccccc}
\hline \hline Object & B & date & Tel + Spectrograph & Exp. Time & $\mathrm{T}_{e f f}$ & $\log \mathrm{g}$ & $v_{t}$ & {$[\mathrm{Fe} / \mathrm{H}]$} \\
\hline BS 16550-087 & 14.56 & June 2005 & SUBARU + HDS & $12 \times 2400 \mathrm{sec}$ & 4750 & 1.4 & 2.3 & -3.50 \\
CS 22873-166 & 12.80 & April 2008 & VLT Kueyen + UVES & $4 \times 5200 \mathrm{sec}$ & 4550 & 0.9 & 2.1 & -2.97 \\
CS 22897-008 & 14.02 & June 2008 & VLT Kueyen + UVES & 5x 5200 sec & 4900 & 1.7 & 2.0 & -3.41 \\
\hline
\end{tabular}

\section{Results}

In Fig. 1 are shown the results of the abundance ratios $[\mathrm{Pd} / \mathrm{Ba}]$ as a function of $[\mathrm{Sr} / \mathrm{Ba}]$. Despite of the use of $10 \mathrm{~m}$ class telescopes and the most advanced spectrographs, only one clear measurement has been possible. For the other two stars, we only obtained upper limits. We gathered also in this diagram results found in the literature and preliminary results for the set of stars used for the Large Programme "First Stars" (private communication).

\section{Discussion}

Our results confirm the correlation between the ratios $[\mathrm{Pd} / \mathrm{Ba}]$ and $[\mathrm{Sr} / \mathrm{Ba}]$. These results can 
be compared with the detailed calculation of a galactic chemical evolution model [12] where the weak and main r-process are assumed to be the stars of $8-10 M_{\odot}$ and $20-30 M_{\odot}$ respectively. The new data seems to favor a slope closer to unity than the one found in their paper. The correlation, close to unity indicates that a significant fraction of the Palladium found in extremely metal poor stars is built by the weak r-process.

As new detailed calculations have been performed for the synthesis of the light neutron-capture elements (see for example [8], [7], [3], [13], [2] and reference therein), it would be very interesting to compute Galactic Chemical Evolution models including these new prescriptions and test them with our new results.

On the observational side, it would be important to continue the search of very metal poor stars with high $[\mathrm{Sr} / \mathrm{Ba}]$ ratios in order to confirm our results. This task definitely requires a large amount of observing time as a large sample of stars needs to be observed in order to identify a few very metal poor stars with high $[\mathrm{Sr} / \mathrm{Ba}]$ abundance ratio .

\section{References}

[1] Alvarez R., Plez B., 1998 A\&A 330, 1109

[2] Arcones \& Montes 2010, submitted to ApJarXiv1007.1275

[3] Farouqi, K., Kratz, K.-L., Pfeiffer, B., Rauscher, T., Thielemann, F.-K., Truran, J. W. 2010 ApJ712,1359

[4] Grevesse, N. \& A. J. Sauval 2000, Origin of Elements in the Solar System. Edited by O. Manuel. p.261

[5] Hill, V. et al. 2002 A\&A 387, 560

[6] Ishimaru, Y., Wanajo, S., Aoki, W., Ryan, S. 2004 ApJ600, L47

[7] Montes, F., Beers, T. C., Cowan, J., Elliot, T., Farouqi, K., Gallino, R., Heil, M., Kratz, K.-L., Pfeiffer, B., Pignatari, M., Schatz, H. 2007 ApJ671,1685

[8] Qian, Y.-Z.; Wasserburg, G. J. 2008 ApJ687, 272

[9] Sneden, C., Cowan, J., Lawler, J., Ivans, I., Burles, S., Beers, T., Primas, F., Hill, V., Truran, J., Fuller, G., Pfeiffer, B., Kratz, K. 2003 ApJ591, 936

[10] Travaglio, C., Gallino, R., Arnone, E., Cowan, J.; Jordan, F., Sneden, C. 2004 ApJ60, 864

[11] Truran, J. 1981 A\&A97,391

[12] Wanajo, S., Ishimaru, Y. 2006 Nucl. Phys. A, 777, 676

[13] Wanajo, S., Janka, H.-T., Mueller, B 2010, ApJ Lett., submitted; arXiv1009.1000 